\title{
The Study on the Development of Private Higher Education based on the view of Non-contiguous Orbital Maneuver
}

\author{
Jianwei Shi, Maohua Li \\ Office of Strategic Planning, Institute for Corporate Social Responsibility, Xi'an Siyuan University \\ Xi’an, China
}

\begin{abstract}
The theory of "S-shaped curve" is of important guiding and practical significance for the transformation of the new and old higher education modes in China. On the one hand, the traditional momentum that supported the growth of higher education in China in the past has moved to the top of the "S-shaped curve." Over the past 40 years of reform and opening up, the traditional scale has been the main driving force for the rapid growth of higher education in China. However, in recent years, the current momentum has begun to move towards the "ceiling" of the "S-shaped curve" and is facing the "inflection point" of the transformation of higher education. If we rely on "scale" to maintain the high-speed growth of the past, it will only lead to the decline of the quality of higher education. This paper traces the track of the development of higher education in China, and further verifies the theory of "S-shaped curve." It is an irreversible rule that the traditional scale will inevitably be weaken when it reaches a particular stage.
\end{abstract}

\section{Keywords-S-shaped curve; Higher education; NOM}

\section{INTRODUCTION}

Private universities are typically not operated by governments, although many receive tax breaks, public student loans, and grants 1 . With globalization and the rise of the knowledge economy, there has been a worldwide increase in demand for higher education (HE) which has resulted in the proliferation of private higher education institutions (PHEIs) (Stander E, Herman C. 2017). Student loyalty is an essential goal of educational institutions due to its various benefits like competitive advantage, providing financial support and influence teaching quality via committed behavior (Meng L L, 2017). Private Universities must apply a new angle of thinking that contains elements of flexibility, speed, innovation, and integration (Hartati S, etc., 2018).

The methodology is the systematic, theoretical analysis of the methods applied to a field of study 2. And research methodology is a systematic way to solve a problem3. To study the development of private higher education based on the view of non-contiguous orbit maneuver, three types of research methodologies which are literature analysis, experience summarization and exploratory study are used.

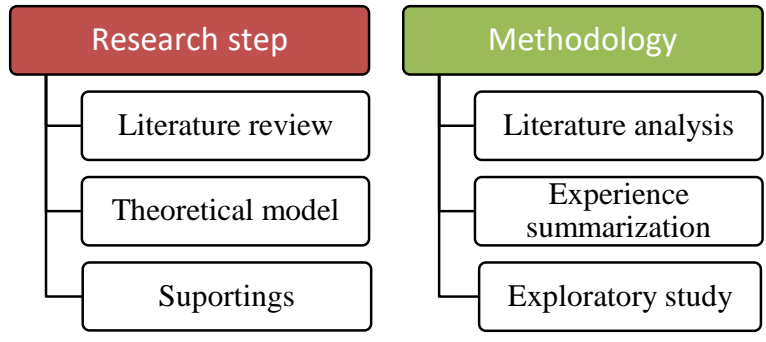

Fig. 1 Research content and methodology

Firstly, this paper uses historical analysis to study the relevant literature which can be seen in the chart above. And then according to the non-contiguous orbital maneuver introduced by Chinese premier Keqiang $\mathrm{Li}$, and with experience summarization, this paper builds a theoretical model for the development of Chinese private higher education. Last but not least, this paper wants to constructive supporting to this conceptual model by the exploratory study.

\section{LITERATURE REVIEW}

At present, the research on connotation development of higher education (CDHE) has been gradually refined, and the connotation of CDHE has been interpreted from multiple dimensions. After summarizing and combing, this paper holds that the current scholars and researchers understand the problems of CDHE from the different aspects. The CDHE is interpreted as promoting the development of higher education by stimulating the initiative of the subject and relying on the strength of the item and optimizing the relationship. For example, Zhang Shenghua pointed out that by optimizing the mode and method of CDHE, teachers' teaching level and research ability can be improved, teachers' vitality can be stimulated, and CDHE can be promoted. Zhang Wei and others advocate the establishment of an endogenous safeguard mechanism through the linkage, cooperation, and coordination among different elements to improve the development of higher education. Some studies have applied connotation development to the field of education management, focusing on issues such as systems, policies, and governance models. Zhang Haojie and others advocate the establishment of operation or the formulation of articles of association to promote the quality of connotation development in private university. These studies have found that people and institutions, organizational structure and other factors play an

\footnotetext{
${ }^{1}$ Scouring: Wikipedia, website: https://en.wikipedia.org/wiki/Private_university

${ }^{2}$ Scouring: Wikipedia, website: https://en.wikipedia.org/wiki/Methodology

${ }^{3}$ Scouring: website: https://arxiv.org/pdf/physics/0601009.pdf.
} 
essential role in promoting higher education, but they have not explored the CDHE from the dimension of development, which has become a partial discussion and improvement on the development of expertise. Furthermore, people's thoughts and behaviors, and their relationships are influenced by culture. Therefore, some studies directly interpret the CDHE as fostering endogenous forces of knowledge through learning, thus promoting education.

For example, Hao Jianhua illustrates how to realize individualized education based on the school's endogenous culture by demonstrating the standard of traditional culture construction in local Primary School. Warring Farmers have just given examples of cultural creation in No. 1 Middle School of Laizhou to prove how to make culture an endogenous driving force for educational development. But learning should play a role through people's thought and behavior, system implementation, and so on. It is not directly related to the CDHE. Besides, the CDHE should be discussed at least from the two dimensions of human and innovation, while the discussion of CDHE on culture is a kind of research that avoids and neglects emphasis.

\section{ABOUT NOM}

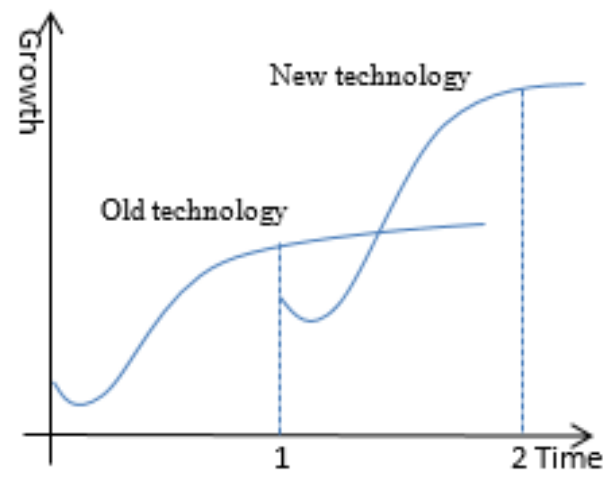

Fig. 2 S-shaped curve of old and new technology

When premier Keqiang Li visited the National Development Institute of Peking University on April 15, Lin Yifu, honorary Dean of the National Development Institute, introduced to the Premier the latest research results of the New Economic Index, which showed that the new economy had been very active recently before the traditional economic recovery. Premier Li mentioned the "S-shaped curve" theory in management and economics: when the old kinetic energy growth is weak, new kinetic energy will emerge, which can support new development (Sourcing: http://www.gov.cn/ xinwen/2016-05/21/content_5075377.htm).

The so-called "S-shaped curve" theory refers to that the growth of each technology is an independent "S-shaped curve." From the graph, it can be seen that the technological progress of a technology in the import period is relatively slow, once it enters the growth period, it will show exponential growth, but when the technology comes the mature period, it will move to the top of the curve, and there will be growth with highest rate. And then it will slow down with lack of motivation. At this time, new technologies will flourish underneath, forming a new "S-shaped curve" and eventually surpassing traditional techniques. Therefore, the transformation of old and new technologies promotes the formation of a peak of continuous technological progress, thus driving the development of the "new economy."

\section{THE THEORETICAL MODEL FOR THE DEVELOPMENT OF PRIVATE HIGHER EDUCATION BASED ON THE VIEW OF NON-CONTIGUOUS ORBITAL MANEUVER}

\section{A. Education assistantship stage developing by the scale}

Education assistantship or Non-academic education refers to all kinds of training, further education and research courses such as training courses, driving schools, postgraduate courses, postgraduate entrance examination and guidance classes, which belong to the category of education assistantship. The students who participate in education assistantship education complete their studies and pass the examinations. The school or training university shall issue the certificate of completion of training, but non-academic education and training shall not be allowed to issue the certificate of degree. Certificates of academic achievement shall not be issued to those who are unable to issue degree certificates, fail to complete the courses, or fail to pass the grades. Compared with academic education, the most significant difference is that the state does not recognize certificates. They may not take part in unified national examinations, such as civil servants, judiciary, accountants, architects, architects, architects, physicians, etc. or take junior college entrance examinations. Some of the graduate students continue to study at the academic level.

\section{B. Development stage for higher vocational education}

Judging from the level of productivity and scientific and technological development in our country, there is still a large shortage of specialized knowledge. The level of product development in China is very unbalanced, and a large number of low-level productivities need to be improved. The transformation rate of scientific and technological achievements in China is low, and there is a shortage of talents engaged in the transformation and application of scientific and technical achievements. Generally speaking, China's economy is in the process of industrialization, commercialization, socialization, and modernization. The history of industrialized developed countries shows that the most significant demand at this stage of development is the application-oriented senior specialized personnel, which is roughly equivalent to the talents trained by our present higher vocational education and more top professional education institutes. For example, the popularity of higher education enrollment in the United States has reached $60-70 \%$ of the age-appropriate population, and the number of community college students at the junior college level accounts for about $50 \%$.

${ }^{4}$ Scouring: http://www.xinhuanet.com/politics/2016-05/22/c_129004800.htm. 


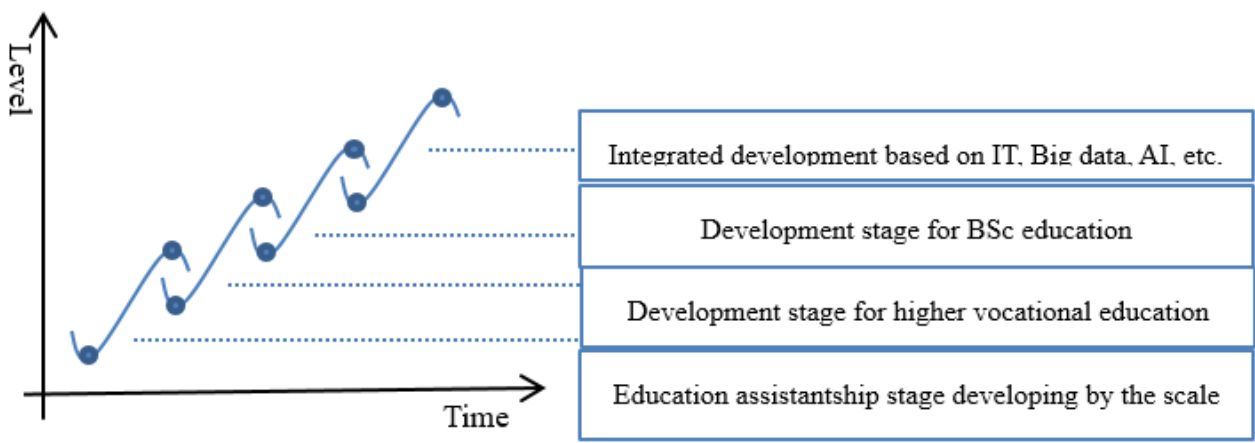

Fig. 3 The historical development of PHE based in based on NOM

To cultivate middle and senior talents with certain professional knowledge and skills; to cultivate talents who can adapt to production, management, service and technology application; to cultivate talents who have certain theoretical knowledge, special skills and can engage in a certain profession or a certain type of work, whose knowledge is taught in the light of "practicality" and "practicality".

\section{Development stage for BSc education}

Undergraduate education (BSc) is the main body of higher education in China, and it plays a central role in the structure of higher education. For a long time, BSc students have accounted for the majority of the total number of higher education students in our country. Only with the popularization of higher education and the expansion of higher vocational and technical education in recent years, the number of specialist students has increased substantially. In 2002, the number of specialist students exceeded the number of BSc students for the first time, and some achievements have been made in structural adjustment for many years. However, from the development trend of higher education in the world, the needs of social and economic development in China and the structure of higher education itself, there are still many problems in this structure.

The length of BSc education is generally four years, and some specialties such as medical science are five years or more. Education at this level includes basic theory education, basic and professional theoretical knowledge and skills education in a specialty or field. Students receiving BSc education carry out experiments, teaching practices and social surveys, receive scientific research training in certain disciplines, write graduation theses and complete graduation projects following the BSc education syllabus and the courses related to planned learning. After completing all the courses prescribed in the teaching plan, those who pass the examination shall be granted graduation, undergraduate diploma, and bachelor's degree.

To cultivate high-level talents who have a solid grasp of the underlying theory, expertise, and necessary skills of the subject and have the preliminary ability to engage in scientific research or undertake specialized technical work. BSc students are required to have a reasonable knowledge structure, master the general methods of scientific work, be able to judge and solve practical problems correctly, have the ability and habit of lifelong learning, and be able to adapt to and be competent in changing occupational fields. The teaching of BSc education knowledge should not only develop to the depth of disciplines but also pay attention to the horizontal relationship between subjects. At the same time, BSc education pays attention to cultivating students' scientific thinking ability, creativity, innovation, and entrepreneurship.

\section{Integrated development based on IT, Big data, AI, etc.}

Talents are not only the key factor to promote the development of IT, Big data, and AI, but also the fundamental force to determine the level of growth in the field of IT, Big data, and AI in a country. It is the responsibility and mission of higher education to cultivate talents related to artificial intelligence. In promoting the integration of higher education and AI, China has not only the aim of revitalizing its traditional advantages but also the consideration of coping with the increasing international competition pressure in related fields. Artificial intelligence (AI) is a technological science that studies the use of advanced digital technology to enable machines to effectively perform highly complex tasks (thinking, reasoning, etc.) like human beings. It is regarded as an essential technology to influence and change the future society. Therefore, it has attracted the attention and promotion of many countries. To seize the strategic opportunities for the development of artificial intelligence and build the first-mover advantages in the field of artificial intelligence, the Chinese government has actively adjusted the thinking of running higher education. On the one hand, it insists that higher education should comply with the new requirements of the era of artificial intelligence and train more talents suitable for the development of artificial intelligence; on the other hand, it puts forward the idea that "technology should be man-made." This principle emphasizes that higher education should have the courage to transcend the demands of the technological era and carry out humanistic knowledge in-depth, to achieve the unity of scientific literacy and humanistic literacy in the construction of talent team

\section{CONCLUSION AND DISCUSSION}

How to get out of a new "S-shaped curve" to drive the transformation of the old and late stages of higher education is the key to the development of higher education in China. To this end, we put forward the following policy recommendations Firstly, the problem of market failure in the import period of "S-shaped curve" is solved. According to the "S-shaped curve" theory, in the process of technological progress, new technology develops very slowly during the introduction period 
and faces uncertainties and risks such as the failure of R\&D, market failure, lock-in of old technology. Therefore, the government cannot adopt the "aid hand" of technological innovation in the way of "flood irrigation." Secondly, efforts should be made to create an ecological environment in which innovation drives the development of "new higher education." One is to inform the transfer of intellectual property rights to the "expressway" to promote the development of "new higher education" in China. Public or state-owned schools have abundant scientific research power, but because of the asymmetric incentives, the enthusiasm of scientific research personnel is not high. Therefore, it is suggested that scientific research personnel should be encouraged to innovate through internal R\&D division and outsourcing $R \& D$ project division, to promote the development of the "new economy." Finally, we should focus on creating an ecological environment of innovation-driven entrepreneurship.

On the one hand, we will continue to vigorously promote the reform of "simplified government and decentralized power" and "commercial system," and promote "mass entrepreneurship and innovation." New and old technologies form two S-shaped curves. The emergence of new technology generally represents the success of a group of modern universities.

\section{ACKNOWLEDGMENTS}

This work is sponsored by the research project of Shaanxi Association of Higher Education. Project No.: XGH19058

\section{REFERENCES}

[1] Osama F. Al-Kurdi, Ramzi El-Haddadeh, Tillal Eldabi. The role of organizational climate in managing knowledge sharing among academics in higher education[J]. International Journal of Information Management,2020,50.

[2] John Michael Dunning. Professional superheroes: Are changes in higher education stretching hospitality management academics professionalism to the limit?[J]. Journal of Hospitality, Leisure, Sport \& Tourism Education,2019,25.

[3] Tine Nielsen, Jesper Dammeyer. Measuring higher education students' perceived stress: An IRT-based construct validity study of the PSS-10[J] Studies in Educational Evaluation,2019,63

[4] Ilaria Stura, Tiziana Gentile, Giuseppe Migliaretti, Enrica Vesce. Accreditation in higher education: Does disciplinary matter?[J]. Studies in Educational Evaluation,2019,63

[5] Aldo Ramirez-Arellano. Students learning pathways in higher blended education: An analysis of complex networks perspective[J]. Computers \& Education, 2019,141.

[6] Luis R. Murillo-Zamorano, José Ángel López Sánchez, Ana Luisa Godoy-Caballero. How the flipped classroom affects knowledge, skills, and engagement in higher education: Effects on students' satisfaction[J]. Computers \& Education,2019,141.

[7] Meng Zhang, Chen-Nan Sun, Xiang Zhang, Phoi Chin Goh, Jun Wei, David Hardacre, Hua Li. High cycle fatigue life prediction of laser additive manufactured stainless steel: A machine learning approach[J]. International Journal of Fatigue,2019,128.

[8] Cristina Carapeto, Daniela Melaré Vieira Barros. Nutrition and health as virtual class at Open University (Portugal): pedagogical strategies for higher education[J]. International Journal of Educational Technology in Higher Education,2019,16(1).

[9] Kyle M. L. Jones. Learning analytics and higher education: a proposed model for establishing informed consent mechanisms to promote student privacy and autonomy[J]. International Journal of Educational Technology in Higher Education,2019,16(1).

[10] Kai Wang, Chang Zhu. MOOC-based flipped learning in higher education: students' participation, experience, and learning performance $[\mathrm{J}]$. International Journal of Educational Technology in Higher Education,2019,16(1).

[11] Nicola Reimann, Ian Sadler, Kay Sambell. What's in a word? Practices associated with 'feedforward' in higher education[J]. Assessment \& Evaluation in Higher Education,2019,44(8).

[12] Michael Henderson, Tracii Ryan, Michael Phillips. The challenges of feedback in higher education[J]. Assessment \& Evaluation in Higher Education,2019,44(8) 